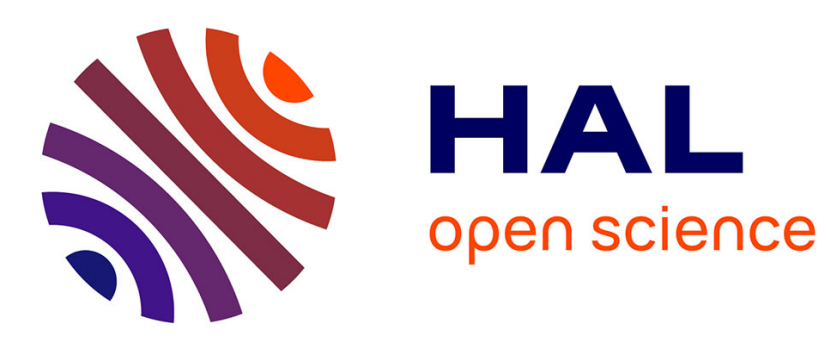

\title{
The continuous modeling of charge-stabilized colloidal suspensions in shear flows
}

Yannick Hallez, Ioannis Gergianakis, Martine Meireles, Patrice Bacchin

\section{To cite this version:}

Yannick Hallez, Ioannis Gergianakis, Martine Meireles, Patrice Bacchin. The continuous modeling of charge-stabilized colloidal suspensions in shear flows. Journal of Rheology, 2016, vol. $60\left(\mathrm{n}^{\circ} 6\right)$, pp. 1317-1329. 10.1122/1.4964895 . hal-01392284

\section{HAL Id: hal-01392284 \\ https://hal.science/hal-01392284}

Submitted on 4 Nov 2016

HAL is a multi-disciplinary open access archive for the deposit and dissemination of scientific research documents, whether they are published or not. The documents may come from teaching and research institutions in France or abroad, or from public or private research centers.
L'archive ouverte pluridisciplinaire HAL, est destinée au dépôt et à la diffusion de documents scientifiques de niveau recherche, publiés ou non, émanant des établissements d'enseignement et de recherche français ou étrangers, des laboratoires publics ou privés. 


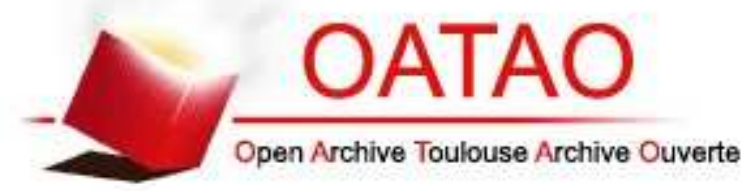

\section{Open Archive TOULOUSE Archive Ouverte (OATAO)}

OATAO is an open access repository that collects the work of Toulouse researchers and makes it freely available over the web where possible.

This is an author-deposited version published in : http://oatao.univ-toulouse.fr/ Eprints ID : 16500

To link to this article : DOI : 10.1122/1.4964895

URL : http://dx.doi.org/10.1122/1.4964895

To cite this version : Hallez, Yannick and Gergianakis, Ioannis and Meireles, Martine and Bacchin, Patrice The continuous modeling of charge-stabilized colloidal suspensions in shear flows. (2016) Journal of Rheology, vol. 60 ( $\mathrm{n}^{\circ}$ 6). pp. 1317-1329. ISSN 01486055

Any correspondence concerning this service should be sent to the repository administrator: staff-oatao@ listes-diff.inp-toulouse.fr 


\title{
The continuous modeling of charge-stabilized colloidal suspensions in shear flows
}

\author{
Y. Hallez, ${ }^{\text {a) }}$ I. Gergianakis, M. Meireles, and P. Bacchin \\ Laboratoire de Génie Chimique, Université de Toulouse, CNRS, INPT, UPS, Toulouse, France
}

\begin{abstract}
Flows of concentrated colloidal suspensions may exhibit a rich set of behaviors due to both hydrodynamic and colloidal interactions between the particles. Colloidal flows are generally modeled with an effective Navier-Stokes equation and a mass balance for the solid phase involving a diffusion coefficient given by the generalized Stokes-Einstein relation. This picture corresponds to a near equilibrium regime in which entropic and colloidal forces dominate over hydrodynamic interactions, the latter being totally ignored. On the other hand, suspension flows far from equilibrium require the modeling of significant hydrodynamic stresses responsible in particular for shear-induced migration, a phenomenon known to occur in some industrial processes involving colloids, such as ultrafiltration. The choice of the proper model ingredients requires a knowledge of the domains in parameter space in which colloidal or hydrodynamic effects are dominant. In this article, such a phase diagram is established for a channel flow of charge-stabilized colloids with a version of the suspension balance model including both colloidal and hydrodynamic effects at the continuous level. It is shown that the classical Péclet number is not sufficient to characterize the flow regime. The phase boundary between the colloidal and hydrodynamic regimes exhibits an original shape explained by the dependence of electrostatic interactions with the colloidal surface charge, and in particular by the phenomenon of ionic condensation. We also show that the phase diagram can be predicted based on the knowledge of a rescaled Péclet number comparing the hydrodynamic stress scale to the bulk modulus of the suspension. The criterion determined here provides important guidelines for the efficient modeling of colloidal flows. http://dx.doi.org/10.1122/1.4964895
\end{abstract}

\section{INTRODUCTION}

Flows of colloidal suspensions are involved in a number of everyday life and industrial applications such as the spreading of paint, slurry transport in pipes, ceramic molding, coating, filtration, or food and beverage processing. An ability to predict and control these flows is essential if colloid-based engineering processes are to be optimized, for example, with a view to reducing energy loss by viscous dissipation in pipe flows, controlling mixing and segregation in particle suspensions, or increasing mass or heat transfer phenomena in complex fluids. The characteristics of suspension flows depend on the hydrodynamic and colloidal interactions between particles, and also on their Brownian motion.

The behavior of suspension flows of Brownian hard spheres is often described in two limits, either close to equilibrium or far from equilibrium. The extent of the departure from equilibrium is measured with the Péclet number $P e$, which can be considered either as a ratio of diffusion and advection time scales, or as a ratio of viscous hydrodynamic and thermal stress scales. Near-equilibrium systems are characterized by $P e \ll 1$ and the far-from-equilibrium "pure hydrodynamic" limit corresponds to $P e \gg 1$.

Due to the presence of solid particles in the fluid phase, suspension flows may differ from single-phase flows both qualitatively and quantitatively. The effect of the solid dispersed phase on the bulk suspension rheology is twofold: the existence of particles and interactions between them increases

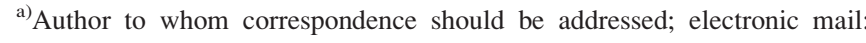
yannick.hallez@univ-tlse3.fr
}

the effective suspension viscosity and can generate potentially anisotropic normal stresses. For $P e \rightarrow 0$, the normal stresses are nearly isotropic and dominated by the contribution of the classical osmotic pressure of the suspension $\Pi$ defined at equilibrium. Being an isotropic stress, the osmotic pressure does not influence the streamlines of the suspension flow. For large Péclet numbers, normal stress differences appear and are commensurate with the average normal stress. The normal stress differences influence the structure of curvilinear flows [1].

Apart from modifying the flow streamlines, the hydrodynamic and colloidal interactions determine mass transport phenomena by generating "shear-induced" and "thermodynamic" mass fluxes, respectively. The thermodynamic flux $\boldsymbol{j}_{\text {th }}$ exists in response to an asymmetry of colloidal interactions around one point, which is translated into the existence of a gradient of osmotic pressure $\Pi$ at this point, so that $\boldsymbol{j}_{\text {th }} \propto-\nabla \Pi$. If the chain rule is employed on this relation, the thermodynamic flux may be written $\boldsymbol{j}_{\text {th }}=-D(\phi) \nabla \phi$, where $D(\phi)$ is the effective gradient diffusion coefficient given by the well known generalized Stokes-Einstein (GSE) relation. Note that this flux only requires the existence of colloidal interactions and a concentration gradient. It therefore exists at any shear rate. Also, $D(\phi)$ is positive for purely repulsive systems, so that colloidal interactions only tend to smooth out any volume fraction fluctuation. The shear-induced mass flux $\boldsymbol{j}_{\mathrm{s}}$ corresponds to the shear-induced migration (SIM) phenomenon [2]. Its form can be constructed either from phenomenological arguments or from the suspension balance model (SBM) but, in both cases, it can be written as the sum of an antidiffusive term proportional to the shear rate gradient and a diffusive term proportional to the shear rate [3-5]. The first term is able 
to create volume fraction gradients and the second one, together with the thermodynamic flux, tends to restore a uniform colloid concentration. This can be responsible for shear banding due to a flow-concentration coupling instability $[6,7]$. Moreover, the intensity of $\boldsymbol{j}_{\mathrm{s}}$ being related to that of the shear rate $\dot{\gamma}$ and of the gradient of shear rate, this flux vanishes when the shear rate vanishes. From this discussion, we understand that $\boldsymbol{j}_{\text {th }} \gg \boldsymbol{j}_{\mathrm{s}}$ near equilibrium and that $\boldsymbol{j}_{\mathrm{s}} \gg \boldsymbol{j}_{\text {th }}$ sufficiently far from equilibrium.

This brief review of possible physical effects in suspension flows can help to shed some light on the various modeling strategies that have been adopted previously. Crossflow filtration is a process in which all these effects have been considered at some point. Although the momentum balance equation of the suspension was generally considered as a (Navier-)Stokes equation with an effective suspension shear viscosity, considerable discussion was involved concerning the modeling of mass transport. It was recognized that considering only a Fickian mass flux with the Stokes-Einstein diffusion coefficient was not sufficient to reproduce available experimental results. Additional phenomena were considered to solve this problem, among them, SIM and the thermodynamic flux described above. On the one hand, it was recognized that including the SIM flux $\boldsymbol{j}_{\mathrm{s}}$ in modeling attempts was necessary for typical microfiltration conditions, i.e., for particles larger than, say, $0.5 \mu \mathrm{m}$ and shear rates of the order of $1000 \mathrm{~s}^{-1}$ [8-11]. On the other hand, considering only the thermodynamic flux $\boldsymbol{j}_{\text {th }}$ in the mass transport equation led to excellent agreement with experimental measurements of bovine serum albumin (BSA) and lactoferrin transport in nanofiltration experiments [12,13]. These macromolecules have a radius of gyration of the order of $3 \mathrm{~nm}$. From a theoretical point of view, nano- and microfiltration are the same flow of a colloidal suspension, but with a Péclet number ranging between $\sim 0$ in nanofiltration and $O\left(10^{2}\right)$ or higher in microfiltration. The published modeling strategies reported here thus perfectly illustrate the aforementioned idea that $\boldsymbol{j}_{\text {th }}$ dominates other fluxes near equilibrium and that $j_{\mathrm{s}}$ is the flux determining mass transport far from equilibrium.

Although it is true that including both fluxes in a model of colloidal flow should always lead to correct solutions, it would be, to date, a somewhat uncontrolled model as the closure relations for both fluxes are still not fully understood. In particular, the thermodynamic flux given by the GSE involves a function depending on many-body hydrodynamic interactions between colloids often in a quite heuristic form, which has a great influence on the diffusion coefficient [14]. It also requires the knowledge of the equation of state (EOS) of the suspension depending on many-body colloidal interactions, the precise determination of which is still an active area of research. On the other hand, the most consistent definition of the SIM flux, based on the SBM [4], involves the divergence of a hydrodynamic stress depending on manybody hydrodynamics, whose form is only known at asymptotic limits, even for hard spheres [15]. It is then of practical importance to determine a priori if a prescribed colloidal flow is "near" or "far" from equilibrium, so that modeling efforts can be concentrated on the one and only relevant flux, if possible. This is the aim of the present work.
For Brownian hard spheres, these "colloidal" (near equilibrium) and "hydrodynamic" (far from equilibrium) regimes are usually characterized by $P e<1$ and $P e>1$, respectively, since the suspension microstructure (determining the rheology) is the result of a balance between the distorting hydrodynamic stress and the restoring thermal stress, the ratio of which precisely defines the Péclet number. It has already been shown that the thermal stresses keep the microstructure of a suspension of hard spheres almost isotropic up to $P e=10$ [16]. In suspensions of "soft" particles (nonhard spheres), the forces helping to restore the microstructure against the flow deformation are of both thermal and colloidal (here electrostatic) origins. The pertinent thermodynamic stress scale is therefore not the thermal scale, especially in concentrated suspensions, and the classical Péclet number thus no longer a relevant dimensionless group, as will be shown in this article. In this work, we will introduce a "dressed" Péclet number $P e^{*}$ as a ratio comparing the hydrodynamic and thermodynamic stress scales, the latter being provided by the EOS of the suspension including both thermal and interaction-induced stresses. The aim of this article is to demonstrate that this number can be computed a priori and used to predict the transition from a colloidal near-equilibrium regime to a hydrodynamic far-from-equilibrium regime in a suspension flow containing charge-stabilized colloids.

To highlight how the flow regime can be anticipated, we will consider the flow of a charge-stabilized colloidal suspension between parallel plates. The model solved in order to predict the concentration and velocity profiles across the channel, accounting for both electrostatic and hydrodynamic effects, is presented in Sec. II. The numerical and analytical computation of a phase diagram identifying the colloidal and hydrodynamic domains in the parameter space will be presented in Sec. III. From these results, we will show that the colloidal and hydrodynamic regimes are determined by $P e^{*} \ll 1$ and $P e^{*} \gg 1$, respectively. A discussion on the main hypotheses of this work and on the conclusions that can be drawn is presented in Sec. IV.

\section{MODEL}

\section{A. The SBM}

Let us consider the fully developed isothermal flow between two flat plates. ${ }^{1}$ The direction of the flow is along the $x$ axis and the direction orthogonal to the plates is the $y$ axis. The volume-averaged momentum and mass conservation equations are written in the SBM formalism [1,4] and, more specifically, an adaptation of the one developed by Frank et al. [15] for Brownian hard spheres. In the fully developed flow, the suspension velocity $\boldsymbol{u}$ is perpendicular to the scalar gradient so the governing equations are

\footnotetext{
${ }^{1}$ The occurrence of shear-induced migration in colloidal suspensions may require high shear rates, especially for small or strongly stabilized colloids, while the viscous dissipation scales as $\dot{\gamma}^{2}$. The isothermal hypothesis might therefore break down in this case. Further comments on this point can be found in the discussion Sec. IV.
} 


$$
\begin{aligned}
& \nabla \cdot \Sigma=0, \\
& \nabla \cdot \boldsymbol{j}=0,
\end{aligned}
$$

where $\Sigma$ is the total bulk stress in the suspension,

$$
\boldsymbol{j}=\phi\left(\boldsymbol{u}^{p}-\boldsymbol{u}\right)
$$

is the particle migration flux relative to the bulk velocity and $\boldsymbol{u}^{\boldsymbol{p}}$ is the particle phase average velocity. The suspension stress can be decomposed into

$$
\Sigma=-P \boldsymbol{I}+\eta \eta_{s}(\phi) \boldsymbol{e}+\Sigma^{s}
$$

where $P$ is a pressure which can contain contributions from the fluid and solid phases but is, in any case, fully determined by the suspension incompressibility condition $\nabla \cdot \boldsymbol{u}=0 . \eta_{s}$ is the suspension relative shear viscosity modeled here as

$$
\eta_{s}(\phi)=1+2.5 \phi(1-\bar{\phi})^{-1}+0.1 \bar{\phi}^{2}(1-\bar{\phi})^{-2}
$$

and depends on the particle volume fraction $\phi$ and the maximum volume fraction $\phi_{m}$ through $\bar{\phi}=\phi / \phi_{m}$. $\boldsymbol{e}$ is the average rate of strain tensor and $\Sigma^{s}$ is the contribution of the solid phase to the bulk stress [17]. In the unidirectional flow considered here, only the projection of Eq. (1) in the $x$ direction is necessary and there is no contribution from the particle stress to the suspension momentum balance. It reads

$$
-\partial_{x} P+\partial_{y}\left(\eta \eta_{s}(\phi) \partial_{y} u\right)=0 .
$$

This equation can be integrated once to yield, with $\dot{\gamma} \equiv \partial_{y} u$ and $\left.\partial_{y} u\right|_{y=0}=0$,

$$
\eta \eta_{s}(\phi) \dot{\gamma}=y \partial_{x} P
$$

The solid phase flux $\boldsymbol{j}$ is determined with the SBM. The particle phase momentum conservation equation reads

$$
\rho\left\langle\boldsymbol{f}_{d}\right\rangle+\nabla \cdot \Sigma^{p}=0
$$

where $\rho$ is the number density of particles, $\rho\left\langle\boldsymbol{f}_{d}\right\rangle$ is the average drag experienced by the particles, and $\Sigma^{p}$ is the particle phase stress [18]. The drag force is given as a function of the slip velocity between the solid and bulk phases as

$$
\rho\left\langle\boldsymbol{f}_{d}\right\rangle=-\frac{9 \eta}{2 a^{2}} f^{-1}(\phi) \phi\left(\boldsymbol{u}^{p}-\boldsymbol{u}\right)
$$

where $f(\phi)$ is the sedimentation hindrance function. Combining Eqs. (3), (8), and (9) leads to

$$
\boldsymbol{j}=\frac{2 a^{2}}{9 \eta} f(\phi) \nabla \cdot \Sigma^{p} .
$$

In the present unidirectional flow, the mass conservation equation (2) then reduces to

$$
\partial_{y}\left[\frac{2 a^{2}}{9 \eta} f(\phi) \partial_{y} \Sigma_{y y}^{p}\right]=0 .
$$

The term in brackets is constant and equal to zero due to the symmetry around the $y=0$ plane. The mass conservation equation is then simply $\partial_{y} \Sigma_{y y}^{p}=0$, or $\Sigma_{y y}^{p}=\left.\Sigma_{y y}^{p}\right|_{y=0}$. A closure relation for the particle phase stress is required to make further progress. We can write this stress as the sum of a thermodynamic part corresponding to the suspension stress at equilibrium, i.e., the osmotic pressure $\Pi$, and a hydrodynamic part designed by Frank et al. to reproduce the correct asymptotic regimes for a suspension of Brownian hard spheres at $P e \ll 1$ and $P e \gg 1$ [15]

$$
\Sigma_{y y}^{p}=-\Pi-\eta \dot{\gamma}\left[b^{-1}(\phi, P e)+c^{-1}(\phi)\right]^{-1},
$$

where

$$
\begin{gathered}
b(\phi, P e)=A \lambda_{2}^{B} P e \phi(1-\bar{\phi})^{-3}, \\
c(\phi)=\lambda_{2}^{H} \eta_{n}(\phi),
\end{gathered}
$$

and here we choose $A=0.4, \lambda_{2}^{H}=0.75, \lambda_{2}^{B}=1.8$, $\phi_{m}=0.64$, and

$$
\eta_{n}(\phi)=0.75 \bar{\phi}^{2}(1-\bar{\phi})^{-2}
$$

in line with the modeling proposed by Frank et al. [15], based on the rheology determined analytically and numerically by Brady and Vicic [19], Phung et al. [20], and Morris and Katyal [21]. In Eq. (12), the term multiplied by $\eta \dot{\gamma}$ is dominated by $b$ at low $P e$ and by $c$ as soon as $P e>1$. When Eq. (12) is used in Eq. (10), the first and second terms lead to the thermodynamic and shear-induced fluxes mentioned in the Introduction, $\boldsymbol{j}_{\mathrm{th}}$ and $\boldsymbol{j}_{\mathrm{s}}$, respectively.

Let us note immediately that the form of the shear viscosity (5) and of the hydrodynamic contribution in Eq. (12) was explicitly designed for hard spheres, neglecting shear-thinning since volume fractions will remain moderate [15,22]. However, in this work we use it even for charge-stabilized suspensions. This modeling is therefore expected to be satisfactory for low surface charges but may be inaccurate for high ones. It is known that the shear viscosity depends strongly on electrostatic interactions, in particular through the secondary electroviscous effect [23]. Increasing the interaction range tends to increase the shear viscosity and the shear-thinning behavior of charge stabilized suspensions at moderate $P e$ numbers (see, e.g., [24] for recent computations). Although many rheological measurements of the shear viscosity of chargestabilized suspensions have been published, and although it is possible to fit these measurements with phenomenological models [25], a general analytical viscosity model depending on the physicochemical parameters (surface charge, interaction range) of the suspension is still elusive even if some results have been obtained in certain asymptotic limits and for electrostatic pair interactions with specific forms [23]. As far as we know, to date, there are no analytical models of the hydrodynamic normal stresses in the presence of colloidal interactions. For the sake of simplicity, we therefore use the original modeling of hydrodynamic effects of Frank et al. for Brownian hard spheres. However, as will become clear at the 
end of this article, the main conclusions drawn are actually linked to the specific behavior of the osmotic pressure in Eq. (12) and are therefore not believed to depend strongly on the precise form of the hydrodynamic stresses.

In using the form (12) for $\Sigma_{y y}^{p}$, we make one additional assumption that is worth underlining. We assume that the anisotropic microstructure generated by the flow does not induce a significant perturbation to the thermodynamic part of the stress. This hypothesis is certainly true for low shear rates but its validity for larger shear rates depends on the type, range, and strength of the physicochemical interactions considered. Comments will be made on this issue in Sec. IV in the light of the results of this work, but in short, it is a justified hypothesis here.

\section{B. The EOS}

The function $\Pi$ in Eq. (12) is the equilibrium EOS of the colloidal suspension. It was computed with different approaches that are briefly summarized here. For uncharged hard spheres, the EOS is very well modeled by [26-29]

$$
\Pi_{\mathrm{HS}}= \begin{cases}\rho k T \frac{1+\phi+\phi^{2}-\phi^{3}}{(1-\phi)^{3}} & \text { for } \quad \phi \leq 0.5 \\ \rho k T \frac{1.85}{\phi_{m}-\phi} & \text { for } \phi>0.5 .\end{cases}
$$

When the colloids are charged, they attract counter-ions near their surfaces, leading to electrostatic interactions due to both the electric field and the pressure exerted by the ions on the surfaces of the colloids. The full, precise computation of these interactions from first principles is still difficult in a general case (nonadditivity of pair-potentials, ion specificity, electrostatic coupling effects, etc.) but satisfactory approaches have been developed especially for the case of 1:1 electrolytes. In this context, the electrostatic coupling between ions and the surfaces is weak, so they can be treated as uncorrelated. If the ions are not too concentrated (say below $0.1 M$ ), their finite size can also be neglected. This picture corresponds to the Poisson-Boltzmann (PB) theory. It is still a nonlinear theory in general. A further simplification is to consider the colloids to be weakly charged and diluted so that the electrostatic potential is lower than $k T / e$ ( $e$ is the electron charge) everywhere. This is the Debye-Hückel theory. In this context, a pair of spherical colloids in an infinite empty medium experiences an interaction potential following the Yukawa form

$$
\beta u=Z^{2} l_{B}\left[\frac{e^{\kappa a}}{1+\kappa a}\right]^{2} \frac{e^{-\kappa r}}{r},
$$

where $\beta=1 / k T, Z$ is the net number of charges on one colloid, $l_{B}=e^{2} / 4 \pi \epsilon k T$ is the Bjerrum length, $\epsilon$ is the liquid dielectric permittivity, $\kappa=\sqrt{8 \pi l_{B} n_{0}}$ is the inverse of the Debye length, and $n_{0}$ is the ion number density in a very large ion reservoir that would be in thermodynamic equilibrium with the suspension. If the interaction potentials are considered additive, the classical integral equation theories can be employed to compute the radial distribution function $g(r)$ of a dispersion, and from it the $\operatorname{EOS} \Pi=\rho k T$
$+\Pi_{\text {contact }}+\Pi_{\mathrm{ES}}$, where $\Pi_{\mathrm{contact}}=\rho k T 4 \phi g(2 a)$ is the pressure contribution due to contacts and

$$
\Pi_{\mathrm{ES}}=-\frac{\rho^{2}}{6} \int r g(r) u^{\prime}(r) d \boldsymbol{r}
$$

is the pressure contribution due to electrostatic interactions. In this article, the Ornstein-Zernike (OZ) equation will be used with a Rogers-Young (RY) closure known to provide accurate results for hard-sphere-Yukawa potentials. If the colloidal surface charge is too high for the Debye-Hückel theory to be valid, the interaction potential can still be written in the form (17) but with effective parameters $Z_{\text {eff }}$ and $\kappa_{\text {eff }}$. When this renormalization procedure is necessary, we employ the method detailed by Trizac et al. [30]. The integral equations theory used with a renormalization approach is valid if the typical surface to surface distance between colloids $d$ is larger than the Debye length, $\kappa d>O(1)$ [31]. This method is used for the simulations of systems with short-ranged interactions, $\kappa a=10$ in this work [see Figs. 1 and 3(a)].

If the double layers overlap significantly (at low salt contents or high volume fraction), the electrostatic forces are no longer pairwise additive. Hopefully, this is the condition in which the cell model is quite precise [32], when the colloids in the suspension feel strong interactions from several neighbors that tend to create a solid-like structure in the dispersion with every colloid in an electro-neutral cell. For simplicity, we suppose that these cells are spherical with the colloid at their center. The one-dimensional nonlinear Poisson-Boltzmann equation can be solved numerically in such a cell to obtain the electrostatic potential field and the ion distribution around one colloid. The electrostatic contribution to the osmotic pressure $\Pi_{\mathrm{ES}}$ is simply related to the total ion density at the boundary of the cell $n(R)$ by

$$
\Pi_{\mathrm{ES}}=n(R) k T-2 n_{0} k T .
$$

More details on the cell model can be found in [30] and [33-35]. For intermediate and long-range interactions, i.e.,

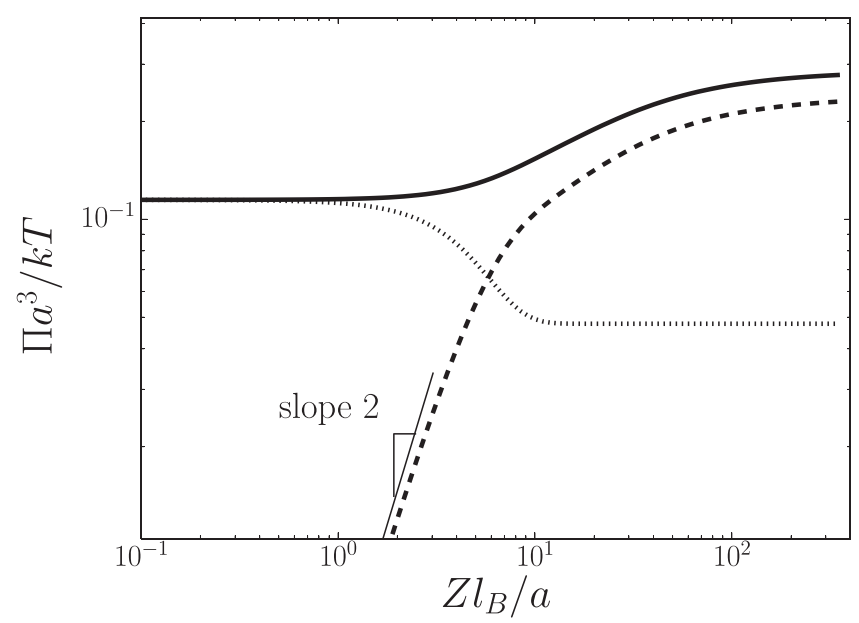

FIG. 1. Calculation of the osmotic pressure $\Pi$ for a volume fraction $\phi=0.2$ and for $\kappa a=10$. Thick continuous line: full pressure $\Pi$; dashed line: electrostatic contribution $\Pi_{\mathrm{ES}}$; dotted line: entropic and contact contributions $\rho k T+\Pi_{\text {contact }}$. Both contributions stem from the equilibrium microstructure predicted with the OZ-RY theory. 
$\kappa a=2$ and $\kappa a=0.5$ in the present work, $\Pi_{\mathrm{ES}}$ will be computed with the cell model. The entropic and contact contributions will be approximated by $\Pi_{\mathrm{HS}}$, which is not true in general since the electrostatic interactions may perturb the radial distribution at contact from its value for hard spheres, as evidenced in Fig. 1 for $\kappa a=10$. The osmotic pressure boils down to $\Pi_{\mathrm{HS}}$ for vanishing surface charges, however, and is dominated by $\Pi_{\mathrm{ES}}$ for large surface charges and longrange interactions, as illustrated in Fig. 2. The approximation $\rho k T+\Pi_{\text {contact }} \simeq \Pi_{\mathrm{HS}}$ is thus only expected to perturb the pressure value slightly in the intermediate charge regime. The EOS obtained for $\kappa a=2$ and $\kappa a=0.5$ are displayed in Figs. 3(b) and 3(c), respectively.

\section{Numerical implementation}

Once the EOS is known for a prescribed set of physicochemical conditions, the final set of equations to be solved for given hydrodynamic conditions is

$$
\begin{gathered}
\eta \eta_{s}(\phi) \dot{\gamma}=y \partial_{x} P, \\
\eta \dot{\gamma}\left[b^{-1}+c^{-1}\right]^{-1}=\left.\Pi\right|_{y=0}-\Pi(\phi) .
\end{gathered}
$$

The group $\eta \dot{\gamma}$ can be expressed as a function of $\phi$ only, thanks to relation (20). When its occurrences in Eq. (21) have all been replaced, the latter equation reduces to a nonlinear algebraic equation for the volume fraction profile $\phi(y)$. This equation is resolved numerically on a 1D grid with standard root finding algorithms available in PythonSciPy. Once the volume fraction profile is known, the shear rate profile is given in Eq. (20) and the velocity profile is obtained by integrating numerically $\dot{\gamma}=\partial_{y} u$. During the numerical resolution, we also impose the constraint

$$
\frac{1}{H} \int_{-H / 2}^{H / 2} \phi d y=\phi_{b},
$$

where $\phi_{b}$ is a prescribed bulk volume fraction set to 0.2 throughout this article.

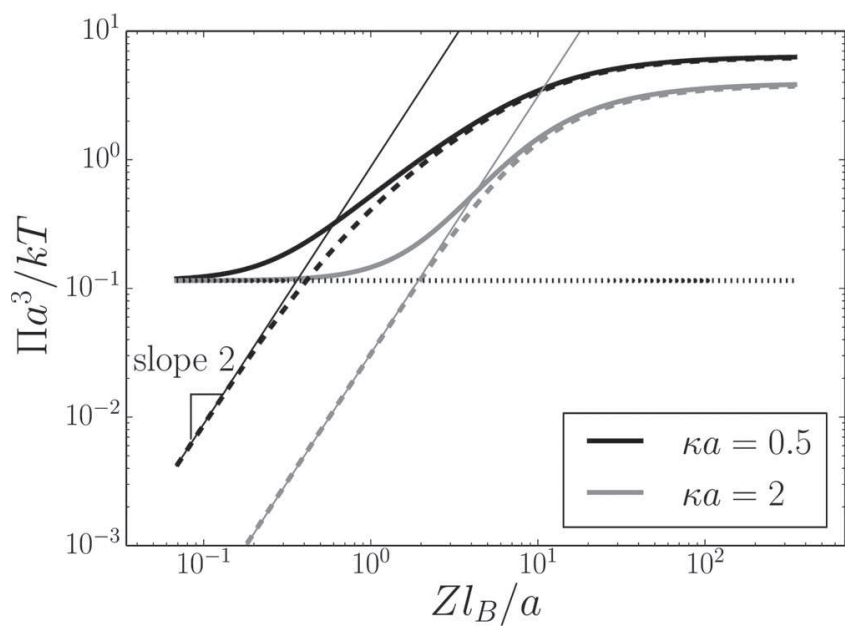

FIG. 2. Same as Fig. 1 but for $\kappa a=0.5$ and 2, and here the electrostatic contribution is given by the cell model and the entropic and contact contributions are taken as $\Pi_{\mathrm{HS}}$, given in Eq. (16). Thin lines: analytical approximation $\Pi_{E S}=\alpha\left(Z l_{B} / a\right)^{2}$ with $\alpha$ given in (29).
In Sec. III, an example of such a computation is presented to highlight the main features of charge-stabilized colloidal flows. Results of many such simulations are then presented and discussed to show how the flow regime can be predicted.

\section{RESULTS}

\section{A. The channel flow of charge-stabilized colloids}

Typical volume fraction and velocity profiles obtained in the fully developed channel flow are reported in Fig. 4 for $P e_{B}=50$ and $\kappa a=2$, and for increasing surface charge densities $\sigma=Z e / 4 \pi a^{2}$. The most spiked curve $(\sigma=0)$ corresponds to the classical flow of a suspension of Brownian hard spheres. The volume fraction profile is fully established when SIM is balanced by Brownian motion and collisions at any point $[15,36]$. The suspension velocity profile is slightly more blunted than the classical channel flow profile due to the shear rheology imposed by relation (5).

When the surface charge density increases, the interparticle repulsions increase and add their contribution to the transverse thermodynamic flux already containing the effects of Brownian motion and collisions. SIM is therefore balanced by a thermodynamic flux of particles that increases with the surface charge density. This is the reason for the flattening of the volume fraction profile observed in Fig. 4(a) with increasing surface charge. Naturally, since a high surface charge tends to restore a uniform concentration profile, it also tends to restore a uniform viscosity and a parabolic flow profile as depicted in Fig. 4(b).

As shown in the example of Fig. 4, it is possible to suppress SIM by increasing the strength of thermodynamic repulsive interactions. The SIM flux scales as $\dot{\gamma} a^{2}$ [2], while colloidal interactions are independent of the shear rate at first order. The appearance of nonhomogeneous concentration profiles due to SIM is thus only-but always - expected for high shear rates. Nonetheless, the shear rate values required to observe SIM in strongly stabilized suspensions, or even for dispersions of small uncharged colloids, might be impossible to attain in practice. The concentration inhomogeneity observed in Fig. 4(a) for the highest surface charge density is quite weak despite a shear rate of the order of $10^{7} \mathrm{~s}^{-1}$. On the other hand, the physicochemical parameters employed are quite realistic and could correspond to moderately charged Ludox HS40 spheres. This certainly explains why part of the literature on the continuous modeling of colloidal flows only considers the effective shear viscosity with the (Navier-) Stokes equation and an effective diffusion coefficient given by the GSE to account for colloidal interactions, neglecting any transverse migration of hydrodynamic origin [12-14]. SIM is however known to occur in some processes involving colloidal suspensions such as crossflow ultrafiltration [8,9,11,37]. One important question for colloidal engineering is then: What are the physicochemical and hydrodynamic conditions required to observe significant effects of either colloidal interactions or SIM? The answer to this question dictates whether efforts need to be made concerning the design of a closure relation for $\boldsymbol{j}_{\text {th }}$ or for $\boldsymbol{j}_{\mathrm{s}}$ (or both). For Brownian suspensions without interactions, the scale of the stress restoring the equilibrium 

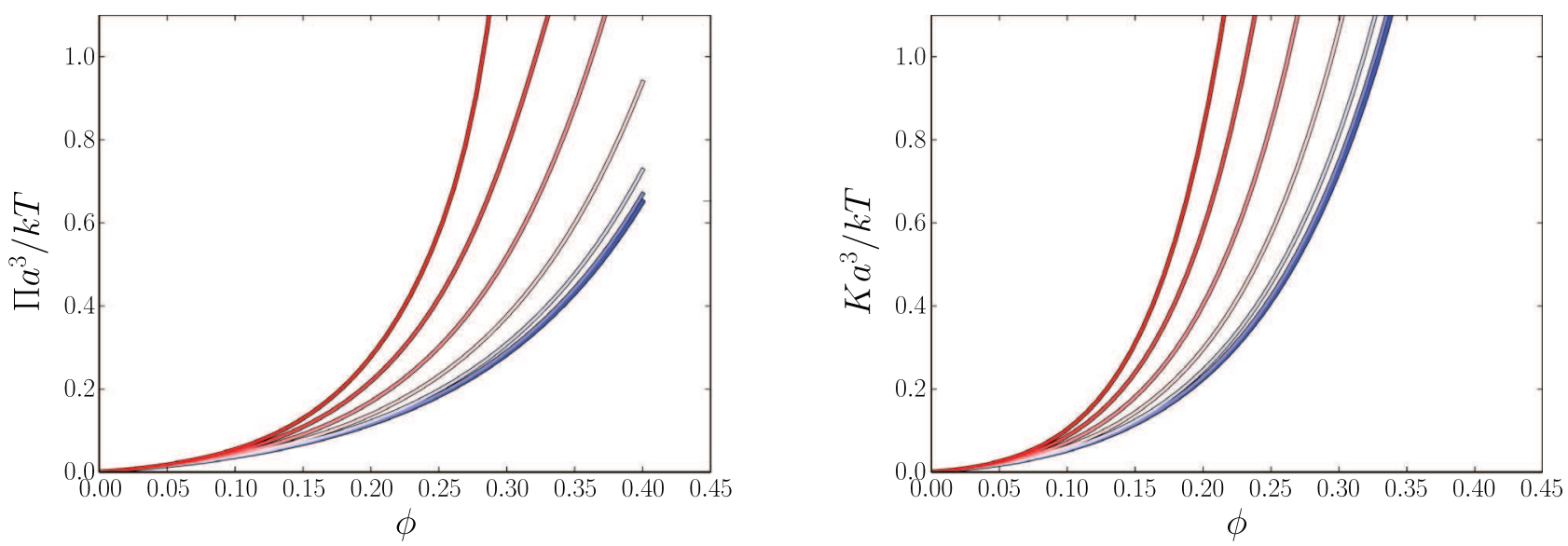

(a) $\kappa a=10$
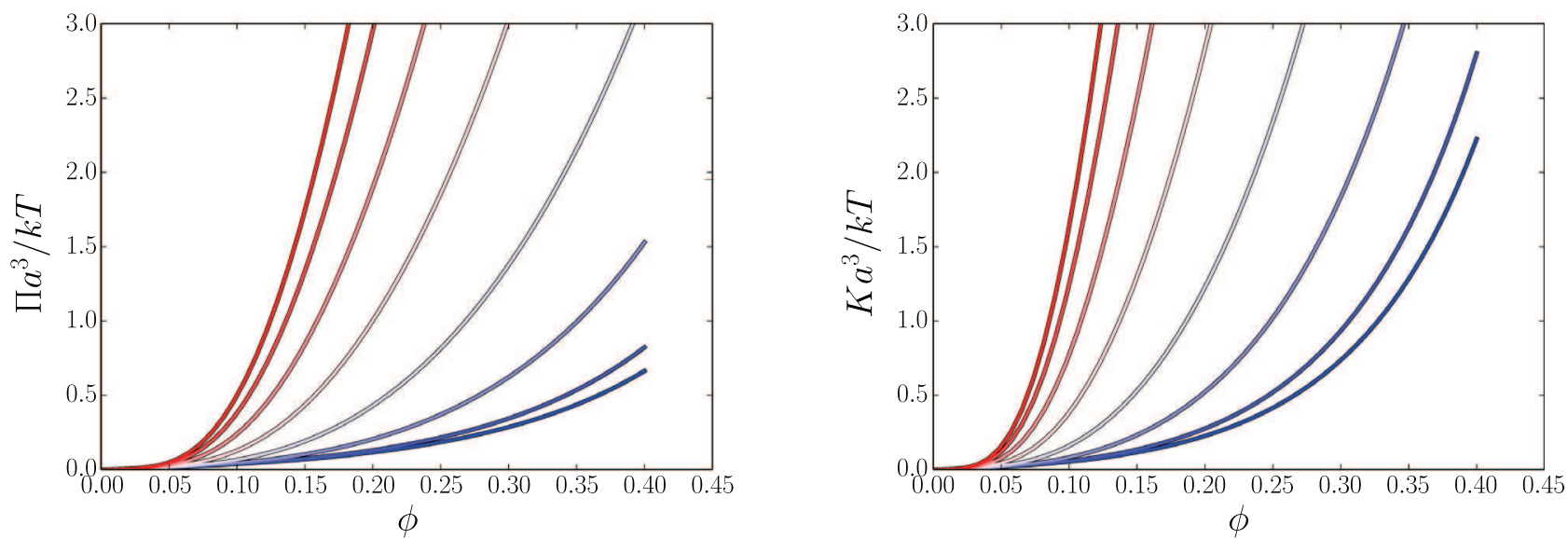

(b) $\kappa a=2$
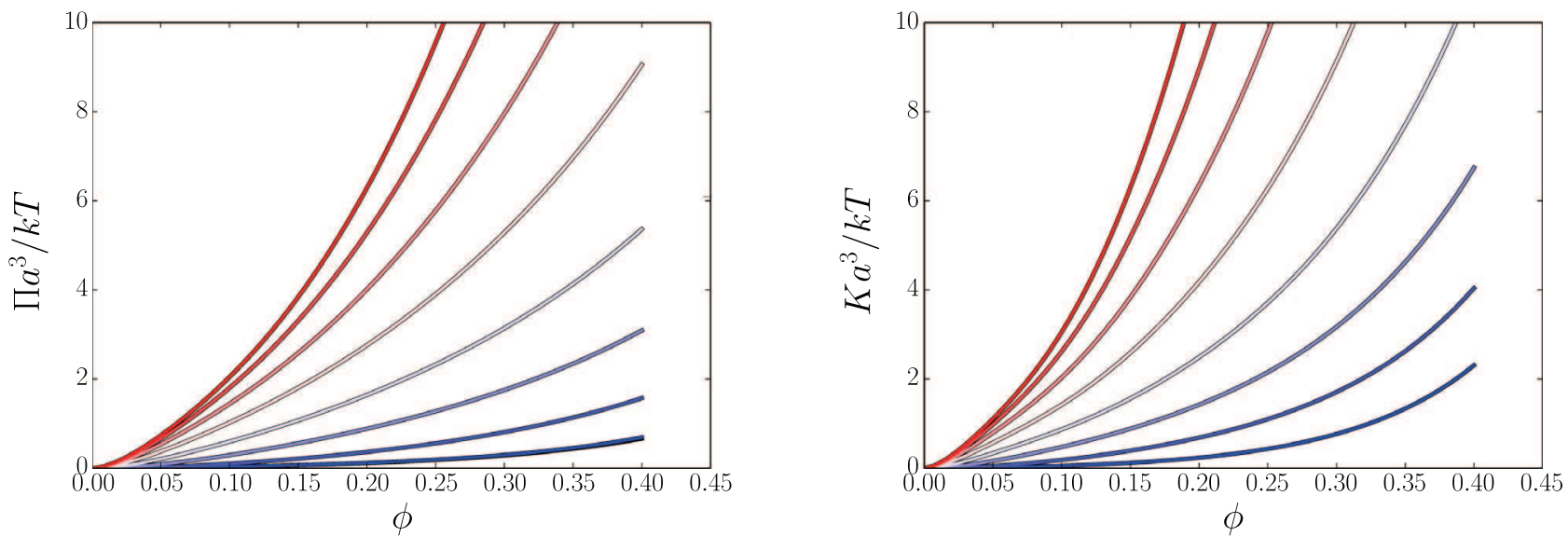

(c) $\kappa a=0.5$

FIG. 3. Scaled osmotic pressure $\Pi a^{3} / k T$ and bulk modulus $K a^{3} / k T=\phi(\partial \Pi / \partial \phi) a^{3} / k T$ used in this article for different interaction ranges and surface charge densities $\sigma$. In each figure, $\sigma=0.001,0.01,0.025,0.050,0.1,0.2,0.5$, and $5 \mathrm{e} / \mathrm{nm}^{2}$ from bottom curves to top curves.

microstructure is of entropic origin, leading to a critical shear rate for observing SIM given by a Péclet number of order unity. For colloidal suspensions dominated by strong hydrodynamics and electrostatics, the restoring stress scale rather depends on the strength and range of electrostatic interactions. The boundary between flows exhibiting SIM and simpler flows therefore lies in a phase space with axes, e.g., $\left(P e, Z l_{B} / a, \kappa a\right)$. The first and second axes can be seen as the dimensionless shear rate and colloidal charge, respectively. The reference scale is given by entropy in both cases. The last axis is relative to the range of interactions. Equivalent axes can be defined by combining the above. 


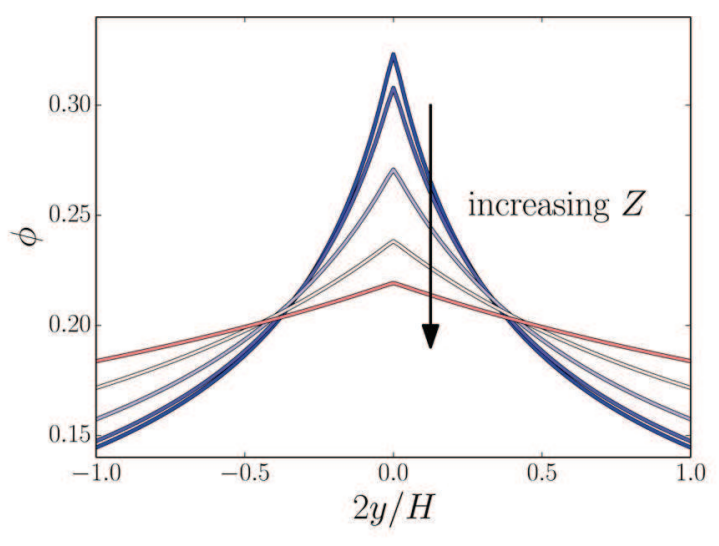

(a) Volume fraction.

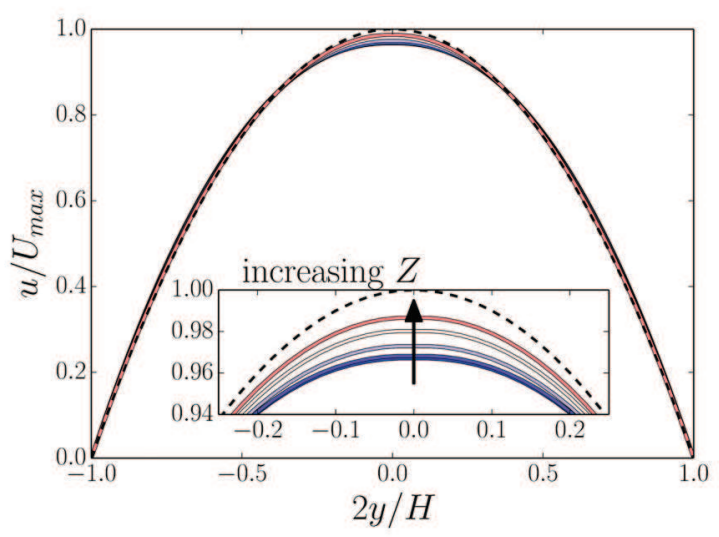

(b) Suspension velocity scaled by the maximum velocity $U_{\max }$ of a parabolic flow (dashed line) with the same flow rate.

FIG. 4. Volume fraction and suspension velocity profiles for increasing surface charge densities $\sigma=0,0.010,0.025,0.050,0.100 \mathrm{e} / \mathrm{nm}^{2}$ at $P e_{B}=50$, $\kappa a=2$, and $a=7.5 \mathrm{~nm}$.

\section{B. The phase diagram}

We now turn to the determination of the phase diagram discriminating the colloidal and hydrodynamic regions in the $\left(P e, Z l_{B} / a, \kappa a\right)$ space. This diagram will be obtained first numerically by solving the model described in Sec. II for various hydrodynamic and physicochemical parameters. It will then be obtained theoretically from arguments developed in this section. This will enable us to shed some light on the peculiar asymptotic behavior of the curve separating the colloidal and hydrodynamic regimes for chargestabilized suspensions.

The colloidal radius was set to $a=7.5 \mathrm{~nm}$. For each couple $\left(\mathrm{Zl}_{B} / a, \kappa a\right)$ determining colloidal interactions, the system (20) and (21) is solved recursively for various $P e$ numbers in order to determine the critical Péclet number $P e_{c}$ such that $\max (\phi) / \phi_{b}=1.1$. This is an arbitrary condition separating the "colloidal" regime with negligible SIM and an almost uniform volume fraction profile $\left(\max (\phi) / \phi_{b}<1.1\right)$ from the hydrodynamic regime characterized by significant volume fraction gradients $\left(\max (\phi) / \phi_{b}>1.1\right)$ due to SIM. The resulting critical points are represented as symbols in the $\left(P e, Z l_{B} / a\right)$ plane shown in Fig. 5 for $\kappa a=0.5,2$, and 10. In aqueous systems, the lower $\kappa a$ value is typical of long-range interactions in semideionized water and the intermediate and

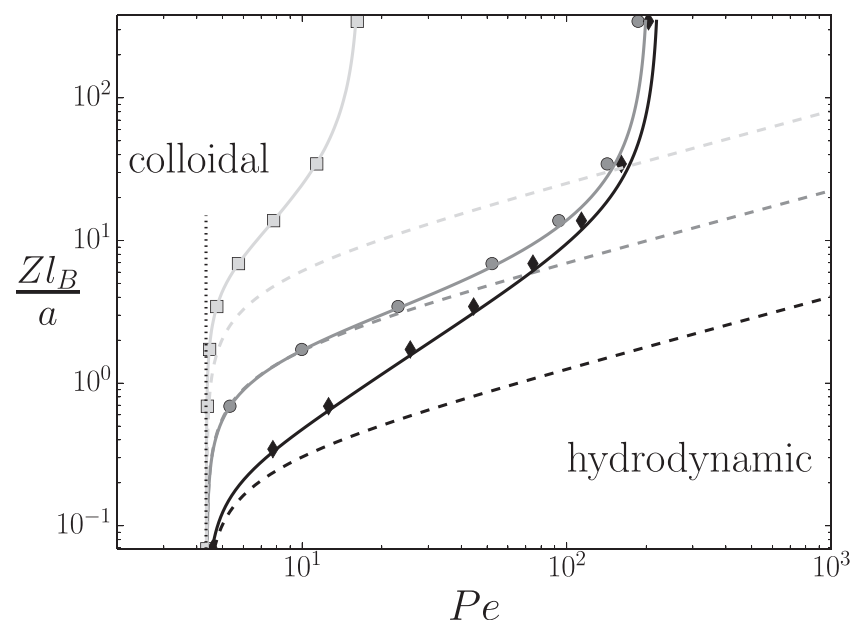

FIG. 5. Phase diagram identifying the colloidal and hydrodynamic domains in the parameter space $\left(P e, Z l_{B} / a, \kappa a\right)$. The colloidal domain is characterized by strong colloidal repulsions preventing SIM. In the hydrodynamic domain, flows exhibit noticeable SIM despite the diffusive effect of colloidal interaction. Symbols: boundary computed numerically with the SBM described in Sec. II. Continuous lines: boundary given by relation (26); dashed lines: boundary given by relation (28); black symbols and lines: $\kappa a=0.5$; gray symbols and lines: $\kappa a=2$; and light gray symbols and lines: $\kappa a=10$.

large values are quite standard for colloids with a size ranging from $10 \mathrm{~nm}$ to $1 \mu \mathrm{m}$, depending on the salt concentration. The range of surface charge density explored corresponds to what would be observable for silica particles, depending on the $p H$. The lowest surface charge density does not affect the dynamics of the colloids, and is representative of a suspension near the isoelectric point. The highest charge considered corresponds to a surface charge density of $5 \mathrm{e} / \mathrm{nm}^{2}$, which is somewhat unrealistically high, but is reported to highlight an asymptotic limit to be described later. The second to last surface charge corresponds to a surface charge density of $0.5 \mathrm{e} / \mathrm{nm}^{2}$, which is classically observed for fully charged silica particles in water $[38,39]$.

The diagram of Fig. 5 shows common features for the three interaction ranges investigated. For all interaction ranges, an S-shaped boundary delimits a colloidal regime at low shear rates and/or high surface charges and a hydrodynamic regime at high shear rates and/or low surface charges. Moreover, all the S-shaped boundaries also exhibit vertical asymptotes in the low charge and high charge regimes. The asymptote in the low charge regime is unique but the location of the asymptote in the high charge regime depends on the interaction range $\kappa a$. Interestingly, the existence of a colloidal regime with no significant evidence of SIM can be observed up to $P e \sim O(100)$ for high surface charges and long-range interactions. It provides support, if it was still required, to the idea that, for charge-stabilized suspensions, the Péclet number is no longer relevant to assess whether mass transport is dominated by hydrodynamic or thermodynamic effects.

Since the boundary in the phase diagram separates regions with dominant thermodynamic effects and dominant hydrodynamic effects, it is tempting to try to define it as the location in the parameter space where some thermodynamic and hydrodynamic stress scales are equal. The local hydrodynamic stress is 
given by the second term in Eq. (12). Since the focus is on flows switching from a hydrodynamic regime to a colloidal regime due to the onset of thermodynamic interactions, we consider flows with $P e>1$, so that the hydrodynamic stress scale is given by the $c$ contribution only and is therefore equal to $\eta \eta_{n}\left(\phi_{b}\right)\langle\dot{\gamma}\rangle \lambda_{2}^{H}$, where $\langle\dot{\gamma}\rangle=U / H$ is the shear rate scale and $U$ is the velocity scale. The thermodynamic stress scale can take different forms. From Eq. (12), it is tempting to use simply the osmotic pressure $\Pi$ but a more precise model can be devised from Eq. (21). Considering this relation at $y=H / 2$, we have

$$
\left.\Pi\right|_{y=0}-\left.\Pi\right|_{y=H / 2}=\left.\left(\eta \eta_{n} \dot{\gamma} \lambda_{2}^{H}\right)\right|_{y=H / 2} .
$$

The boundary between the hydrodynamic and the colloidal regimes is determined by

$$
\max (\phi)=\phi(y=0)=1.1 \phi_{b} .
$$

Since the perturbation from the uniform volume fraction profile is weak, the latter is close to linear [see Fig. 4(a)] so that on the boundary between the flow regimes $\phi(y=H / 2)$ $\simeq 0.9 \phi_{b}$. Taylor expansions of $\Pi$ around $\phi_{b}$ yield

$$
\Pi\left(1.1 \phi_{b}\right)-\Pi\left(0.9 \phi_{b}\right)=\left.0.2 \phi_{b} \frac{\partial \Pi}{\partial \phi}\right|_{\phi_{b}}+O\left(\left(0.1 \phi_{b}\right)^{3}\right)
$$

where $\phi_{b} \partial \Pi /\left.\partial \phi\right|_{\phi_{b}}$ is the bulk modulus $K$, or the inverse of the osmotic compressibility $\chi$, taken at the bulk volume fraction $\phi_{b}$. Equation (23) then suggests that the boundary between colloidal and hydrodynamic regimes is given by a stress balance reading

$$
0.2 K\left(\phi_{b}\right) \simeq \eta \eta_{n}\left(\phi_{b}\right)\langle\dot{\gamma}\rangle \lambda_{2}^{H}
$$

Multiplication of this relation by the entropic stress scale $6 \pi a^{3} / k T$ leads to

$$
P e=c \frac{0.2 \tilde{K}\left(\phi_{b}\right)}{\lambda_{2}^{H} \eta_{n}\left(\phi_{b}\right)}
$$

where $c$ should be an $O(1)$ constant and where $\tilde{K}$ $=K 6 \pi a^{3} / k T$ is a dimensionless bulk modulus. Using relation (15) for $\eta_{n}$ and the EOS determined as described in Sec. II, we predict from Eq. (26) the boundaries represented as continuous lines in Fig. 5. By matching the curves with the numerical results at $Z=0$, the constant $c$ was found to be 0.6 for the bulk volume fraction used in this work. More comments about this value will be made later. As shown in Fig. 5, relation (26) provides very good estimate of the S-shaped boundary for all the interaction ranges and charges investigated. Interestingly, this relation only requires the normal viscosity and the EOS of the colloidal suspension to be known. The latter can be determined numerically as in the present work or experimentally from osmotic compression, or light or neutron scattering measurements. One of the main practical conclusions of this work is therefore that obtaining a diagram like the one of Fig. 5 is easily possible using relation (26) with classical measurements or numerical models of EOSs for a given colloidal suspension, and that it can be done in order to design a flow model as well-suited as possible to anticipated flow conditions.

Regardless of the practical point of building diagrams like the one presented in Fig. 5, the form of the S-shaped boundaries is of interest and may seem counter-intuitive at first glance. In order to understand this form, it is convenient to recall that the osmotic pressure can be decomposed as $\Pi=\rho k T+\Pi_{\text {contact }}+\Pi_{\mathrm{ES}}$, where $\Pi_{\text {contact }}$ arises from collisions between colloids and is related to the pair distribution function at contact, while $\Pi_{\mathrm{ES}}$ is the contribution of electrostatic interactions here, and more generally to any type of interaction at a distance.

At vanishing surface charge density, $\Pi_{\text {contact }} \gg \Pi_{E S}$ and the osmotic pressure is $\Pi(\phi)=\Pi_{\mathrm{HS}}(\phi)+\epsilon\left(\phi, \kappa a, Z l_{B} / a\right)$ where $\epsilon$ is small compared to the other terms and vanishes with $Z$. The boundary equation (26) thus becomes independent of $Z$ and $\kappa a$ at this limit, leading to the vertical asymptote observed in Fig. 5 and determined by

$$
P e=c \frac{0.2 \tilde{K}_{\mathrm{HS}}\left(\phi_{b}\right)}{\lambda_{2}^{H} \eta_{n}\left(\phi_{b}\right)},
$$

where $K_{\mathrm{HS}}=\phi \partial \Pi_{\mathrm{HS}} / \partial \phi$.

For nonzero but still moderate surface charges, the colloidal interactions may be strong enough to alter the osmotic pressure significantly. For the purely repulsive system considered here, the osmotic pressure increases monotonically with the surface charge. The critical shear rate or Péclet number necessary to observe hydrodynamic effects must therefore also increase monotonically with $Z$. This explains the positive and finite slope of the S-shaped boundaries in the intermediate charge regime. A crude attempt at predicting the boundary is possible in the weak charge regime. Both the form of the pair potential (17) and the analytical solution of the cell model in the Debye-Hückel limit suggest an electrostatic contribution to the osmotic pressure $\Pi_{\mathrm{ES}}$ scaling as $Z^{2}$, and this is verified in Figs. 1 and 2. The thermodynamic stress $K$ then derives from a pressure $\Pi_{c o l}+\alpha\left(Z l_{B} / a\right)^{2}$, where $\Pi_{c o l}=\rho k T+\Pi_{\text {contact }}$ and $\alpha$ is a constant that will be commented later. The location of the separation between the colloidal and hydrodynamic regimes is then expected to be given by

$$
P e=c \frac{0.2\left[\tilde{K}_{c o l}\left(\phi_{b}\right)+\left.\phi_{b} \frac{\partial \tilde{\alpha}}{\partial \phi}\right|_{\phi_{b}}\left(\frac{Z l_{B}}{a}\right)^{2}\right]}{\lambda_{2}^{H} \eta_{n}\left(\phi_{b}\right)},
$$

where $\tilde{\alpha}=\alpha 6 \pi a^{3} / k T$ and $K_{c o l}=\phi \partial \Pi_{c o l} / \partial \phi$. This equation is represented with dotted lines in Fig. 5, assuming $\Pi_{\text {col }} \simeq \Pi_{\mathrm{HS}}$. At vanishing surface charge $Z$, we recover relation (27) valid for hard spheres. For $\kappa a=0.5$ and 2, the theoretical prediction from the linearized cell model was employed for $\alpha$

$$
\begin{aligned}
\frac{\alpha}{2 n_{0} k T}= & 2(\kappa a)^{2}\left[(\kappa R+1)(1-\kappa a) e^{\kappa(a-R)}\right. \\
& \left.+(\kappa R-1)(1+\kappa a) e^{-\kappa(a-R)}\right]^{-2}
\end{aligned}
$$


where $R=a \phi^{-1 / 3}$ is the radius of the cell. For $\kappa a=10$, the value of $\alpha$ was measured on the numerical data presented in Fig. 1 in the low charge regime. Note that the model (28) and (29) requires a linearized PB equation, which is valid at high $\kappa a$ and low charges, and a solid-like structure to employ the cell model, which is valid at low $\kappa a$ and high charges. This is why it works best at intermediate $\kappa a$ and $Z l_{B} / a$ values in Fig. 5, and this is also why it is rather useless in practice.

The deficiency of model (28) and (29) does, however, have the merit of showing that, even when it gives satisfactory results as in the $\kappa a=2$ case for intermediate charges, it can definitely not capture the vertical asymptote at high surface charge. Any modeling of the electrostatic contribution to the osmotic pressure as a power law of $Z$ would generate inclined asymptotes like the dashed curves of Fig. 5. The vertical asymptotes obtained numerically are signatures of the thermodynamic stress becoming independent of $Z$ at high surface charge. The analytical model (26) contains this feature, thanks to the modeling of the osmotic pressure detailed in Sec. II B. The reason for the saturation of the pressure at high surface charge is the phenomenon of ionic condensation [31]: when the surface charge is increased toward very high values $\left(Z l_{B} / a>O(10-100)\right)$, counter-ions are increasingly attracted into a small region near the surface of the colloids in a manner such that any new charge added to the surface of the colloid is neutralized by a new "condensed" counter-ion. Consequently, we can define an effective colloid encompassing the real colloid and the condensed ionic region, and attribute an effective charge $Z_{\text {eff }}$ to it. At low surface charges, there is no condensation and $Z_{\text {eff }} \simeq Z$. At high surface charges the effective charge saturates and reaches a finite value $Z_{\text {eff }}^{\text {sat }}(\phi, \kappa a)$ [40]. In consequence, the electrostatic contribution to the osmotic pressure also saturates at an asymptotic value $\Pi_{E S}^{\text {sat }}$ which depends only on $\phi$ and $\kappa a$ as illustrated in Figs. 1 and 2. Since electrostatic interactions become independent of the bare charge $Z$, so does the radial distribution function and the collision contribution also saturates at a value $\Pi_{\mathrm{HS}}^{\mathrm{sat}}(\phi, \kappa a)$. The thermodynamic stress scale $K$ then derives from the osmotic pressure $\Pi^{\mathrm{sat}}(\phi, \kappa a)=\Pi_{\mathrm{col}}^{\mathrm{sat}}+\Pi_{\mathrm{ES}}^{\mathrm{sat}}$. The equation of the boundary in the phase diagram (26) then becomes

$$
P e=c \frac{0.2 \tilde{K}^{\mathrm{sat}}\left(\phi_{b}, \kappa a\right)}{\lambda_{2}^{H} \eta_{n}\left(\phi_{b}\right)},
$$

where $K^{\text {sat }}=\phi \partial \Pi^{\text {sat }} / \partial \phi$. It is a vertical asymptote in the $\left(\mathrm{Pe}, \mathrm{Zl}_{B} / a\right)$ plane since it no longer depends on $\mathrm{Z}$, but its position still depends on the interaction range $\kappa a$, as observed in Fig. 5. It is important to emphasize at this point that a modeling of the EOS based on pair potentials with a classical DLVO or Yukawa form ignoring ion condensation effects would not permit the S-shaped boundary to be recovered on the flow diagram 5. It would only yield boundaries similar to the dashed lines.

Instead of defining a complex boundary in the $\left(P e, Z l_{B} / a\right)$ plane, relation (25) also suggests the definition of a dressed Péclet number,

$$
P e^{*}=\frac{\eta \eta_{n}\langle\dot{\gamma}\rangle}{K}=\eta \eta_{n}\langle\dot{\gamma}\rangle \chi
$$

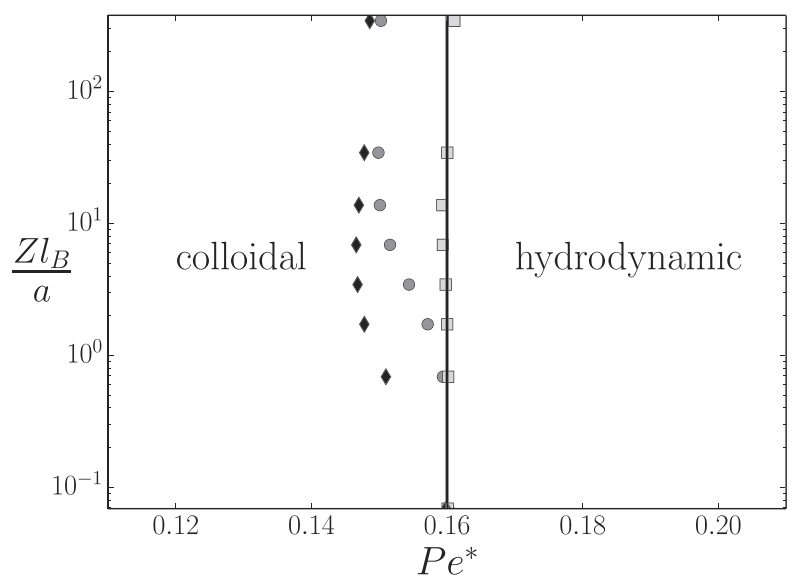

FIG. 6. Phase diagram identifying the colloidal and hydrodynamic domains in the parameter space $\left(P e^{*}, Z l_{B} / a, \kappa a\right)$. Symbols and lines: same as Fig. 5.

equal to an $O(1)$ constant at the transition between the colloidal and hydrodynamic regimes, independently of the separate values of the shear rate, colloidal interaction range, and surface charge. The location of the flow regime boundaries determined numerically with the SBM and represented in the $\left(P e, Z l_{B} / a\right)$ plane in Fig. 5 are recast in the $\left(P e^{*}, Z l_{B} / a\right)$ plane in Fig. 6. As expected, they collapse to a single line with equation $P e^{*}=P e_{c}^{*}$, where $P e_{c}^{*}$ is the (constant) critical dressed Péclet number at the transition between the colloidal and hydrodynamic regimes. At the hard sphere limit, we determined previously that $P e_{c}^{*} \equiv 0.2 c / \lambda_{2}^{H}=0.16$ for the bulk volume fraction $\phi_{b}=0.2$ used in this work. The numerical determination of $P e_{c}^{*}$ for any surface charge and from the SBM results presented in Fig. 6 leads to $P e_{c}^{*}=0.155$ on average, in perfect agreement with the value determined in the hard-sphere regime. All the values of $P e_{c}^{*}$ are found between 0.147 and 0.161 for the full extent of interaction ranges and surface charge investigated in this article while, as mentioned before, they correspond to the full range of realistic values in aqueous suspensions. The idea that the transition between colloidal and hydrodynamic regimes occurs at a constant $P e^{*}$ is therefore quite robust.

To conclude, besides plotting a flow diagram with relation (26) it is also possible to compute the value of the rescaled Péclet number $P e^{*}$ with relation (31) for prescribed hydrodynamic and physicochemical conditions and compare it to unity to determine a priori if the flow is in the colloidal or hydrodynamic regime.

\section{DISCUSSION}

In this article, we have presented a modeling strategy aimed at simulating flows of charge-stabilized colloidal suspensions. The main conclusions are that (i) the Péclet number is not a relevant dimensionless group to characterize the departure from equilibrium in a suspension stabilized by significant electrostatic interactions; (ii) it is possible to define a more relevant rescaled Péclet number as the ratio of viscous and thermodynamic stress scales; (iii) the thermodynamic stress scale, considered here as the bulk modulus at equilibrium, has to be modeled carefully to account for subtle 
physicochemical effects that can influence the flow regime, such as ion condensation. In this section, we propose a few comments to discuss the general validity of these conclusions and the limits of the numerical modeling approach.

\section{A. Comments on the model}

The first critical point concerning the computation of the channel flow with the SBM is probably related to the form of Eq. (12). One of the main assumptions here is that the thermodynamic component of the total stress is isotropic and equal to the osmotic pressure at equilibrium. It is a quite reasonable premise near-equilibrium but it may be wrong at high shear rates. Even though simulations have been conducted at $P e$ numbers as high as $O(100)$, it should now be clear that only $P e^{*}$ is relevant to measure the distance to equilibrium in charge-stabilized suspensions and that, by design, the present simulations are all at $P e^{*} \sim 1$ ( 0.155 on average to be more precise). It would be tempting to use the SBM as formulated in this article for higher shear rates. However, in this case, the flow would make the microstructure of the suspension significantly anisotropic. Since the osmotic pressure of the suspension derives from its microstructure, we could expect deviations between the nonequilibrium thermodynamic component of the suspension stress and the equilibrium osmotic pressure. Additionally, the anisotropy would suggest the use of a thermodynamic stress tensor instead of a single pressure. To date, we know of no analytical model of the thermodynamic stress tensor in a flow of charged stabilized colloids at $P e^{*}>1$, and as a function of $\phi, P e, \kappa a$, and $Z l_{B} / a$. This issue is a cornerstone for the improvement of the continuous modeling of colloidal flows, and it ought to receive attention in future rheological studies. For example, in a very dilute suspension with a typical interparticle distance $\kappa d \gg \kappa a \gg 1$, the SIM flux would scale as $\kappa^{-2} \dot{\gamma}$ instead of $a^{2} \dot{\gamma}$. This effect cannot be obtained with the present equilibrium modeling of the thermodynamic stress tensor, which is more adapted to electrostatically concentrated suspensions.

The second issue with the model also concerns the form of the thermodynamic stress in Eq. (12). Assuming that a flow is considered at $P e^{*}<1$, as in the present work, so that a "simple" equilibrium osmotic pressure can represent the thermodynamic stress tensor reliably, the EOS calculated here are only approximations of the real ones although we took care to use renormalization, the OZ theory, and the cell model in the range of parameters in which they should work best. The most detailed computations of the osmotic pressure at equilibrium require both colloids and ions to be included in a molecular dynamics (MD) or Monte Carlo (MC) simulation box and imply a wait of a few hours or days for statistics to converge. Generating such data with a view to using it for flow modeling is currently unimaginable. Indeed, even simple flows might involve variations of colloid concentration, ion concentration, and even $p H$ making the parameters $\phi$, $\kappa a$, and $Z l_{B} / a$ nonuniform in space and time. Establishing the full dependence of the EOSs in this parameter space would require thousands of such simulations. This illustrates the need for fast, efficient ways of computing EOS of charge-stabilized suspensions with simple models like the ones employed in this article. The development of fast models going beyond the PB theory, like the modified PB theory, density functional theory, or self-consistent field theory, is still a subject of research. Despite the above-mentioned reserves about the precise form of the EOS employed here, we insist on the fact that the EOSs used during the simulations and for the analysis of Sec. III were the same, so the simulations and the conclusions drawn in this work are selfconsistent. In particular, the important idea that ionic condensation and effective charge saturation has to be accounted for is robust and would be obtained with any fully detailed thermodynamic model.

One last limitation of the present modeling strategy is the assumption of isothermal flow. Indeed, reaching $P e^{*}=1$ for small or strongly interacting colloids requires very large shear rates. Since the viscous dissipations scales as $\eta \dot{\gamma}^{2}$, the temperature might increase significantly on experimental time scales. An order of magnitude analysis shows that suspensions of very small colloids with a radius of the order of $10 \mathrm{~nm}$ or less would boil in a few seconds if the shear rate were high enough to observe SIM. The physics described in this article are clearly not relevant in this case. Such shear rates are however so high that they are actually unlikely to be encountered in real applications and these suspensions are in practice simply bound to remain in the $P e^{*}<1$ regime. At the other limit of the colloidal domain, suspensions of particles approaching the micron size do not undergo any significant heating on very long time scales when $P e^{*} \sim 1$, independently of their potential colloidal interactions, so that the isothermal approach is clearly valid. Finally, we consider a suspension of charged-stabilized silica spheres (effective surface charge $\sim 0.05 \mathrm{e} / \mathrm{nm}^{2}$ ) in the intermediate size range. SIM is obtained at higher shear rates for higher values of the osmotic pressure, the latter depending in particular on $\phi$ and the added salt concentration $I$. If we consider somewhat arbitrarily that a temperature elevation of $1 \mathrm{~K}$ in $100 \mathrm{~s}$ is the limit for an isothermal description of the physics, we find that colloids should be larger than a few tenths of nanometers to allow for an isothermal modeling if $I=10^{-2} \mathrm{M}$. For partially deionized suspensions with $I=10^{-3} \mathrm{M}$, the radius of colloids should be at least of the order of $100-200 \mathrm{~nm}$ for the isothermal modeling to be valid. The present work is therefore strictly speaking restricted to suspensions of rather large colloids, with a radius of at least, say, $100 \mathrm{~nm}$, keeping in mind this scale depends on colloidal interactions. Modeling SIM for smaller colloids, or colloids with stronger interactions, would require a nonisothermal approach. This can be achieved by supplementing the momentum and mass conservation equations (1) and (2) with an energy conservation equation and by accounting for the temperature dependence of viscosities.

\section{B. Comments on the main results}

The definition of a new Péclet number as $P e^{*}=\eta \eta_{n} \dot{\gamma} / K$ for colloidal suspensions is one of the main conclusions of this work. Although other rescalings have been proposed in 
the literature for more or less similar purposes, the present definition has some specificities that are worth underlining.

Brady and Vicic [19] proposed a Péclet number defined as $\overline{P e}=\dot{\gamma} a^{2} / D_{0}^{s}(\phi)$ in a scaling theory aimed at modeling the stresses in Brownian, homogeneous, concentrated hard sphere suspensions at low $P e$. Their technique consists of replacing the Stokes-Einstein diffusivity $D_{0}$ (valid in the dilute limit) in the classical $P e$ by the short-time self-diffusivity $D_{0}^{s}$ in concentrated suspensions. The $P e^{*}$ defined here is obtained by replacing the thermodynamic stress $k T / a^{3}$ (valid in the dilute limit) by the bulk modulus deriving from the full osmotic pressure relevant for "electrostatically concentrated" suspensions. The parallel stops here, however, since the $\overline{P e}$ of Brady and Vicic is a microscopic Péclet number used for comparing time scales driving the microstructure distortion in a sheared homogeneous suspension, while $P e^{*}$ is macroscopic in the sense that it is intended to compare the macroscopic thermodynamic and shear-induced mass fluxes in nonhomogeneous suspensions. Moreover, the shorttime self-diffusion coefficient reduces to $D_{0}$ without hydrodynamic interactions so that $\overline{P e}$ reduces to $P e$ in this case, while on the other hand $P e^{*}$ involves the thermodynamic contribution $K$ so that $P e^{*}$ is different from $P e$ as soon as nonhydrodynamic forces are involved.

To compare macroscopic fluxes, it might be tempting to define an effective $P e$ by comparing the thermodynamic diffusive time scale $a^{2} / D_{\nabla}$, where the diffusion coefficient is given by the GSE, and some hydrodynamic time scale associated with SIM, typically $\dot{\gamma}^{-1}$. The rescaled Péclet number would be $\dot{\gamma} a^{2} / D_{\nabla}$, by analogy with the usual Péclet number $\dot{\gamma} a^{2} / D_{0}$. However, this is inappropriate from a simple pragmatic point of view since $D_{\nabla}$ contains the hydrodynamic function $f(\phi)$ while, in the present steady-state channel flow, there is no need to introduce it in the final model (20) and (21). The explanation of this apparent paradox is as follows. The Péclet number is indeed a ratio comparing the strength of hydrodynamic and diffusion phenomena. However, it usually compares an advection flux $\boldsymbol{j}_{a}=\boldsymbol{u} \phi$ and a diffusion flux in a mass transport equation, or advection and diffusion probability fluxes in the Smoluchowski equation for homogeneous sheared suspensions [41]. In the present flow, the mass flux of hydrodynamic origin is not the advection flux. It is the SIM flux $\boldsymbol{j}_{s}$ stemming from many-body hydrodynamic interactions and not from simple advection. This flux contains the same hydrodynamic function $f(\phi)$ as the thermodynamic flux as shown in Eqs. (10) and (12), so that comparing their scales leads to $P e^{*}$, independently of the hydrodynamic hindrance function. For completely general flows, the numbers $P e^{*}$ and $\dot{\gamma} a^{2} / D_{\nabla}$ are thus independent of each other, and both are necessary. The first one compares thermodynamic diffusion and SIM fluxes while the second one compares thermodynamic diffusion and advection fluxes.

Some works on charge-stabilized colloidal flows also introduce the number $A=F a / k T$ comparing the interparticle (here electrostatic) force scale $F$ to the Brownian force scale $k T / a[23,42]$. The ratio $P e / A$ compares hydrodynamic forces to thermodynamic forces in a manner similar to our $P e^{*}$ comparing hydrodynamic and thermodynamic stresses. There is, however, a difficulty in computing the force scale $F$ since it varies between 0 and $\infty$ depending on the distance between two colloids, if we assume it derives from a Yukawa potential (17). The correct force scale depends on some typical interaction distance $L$, which is, a priori, unknown unless the microstructure is computed. On the other hand, we have direct access, with a good precision, to the osmotic pressure in electrostatically concentrated suspensions with the cell model, independently of the knowledge of the microstructure of the suspension. Therefore, even if $P e^{*}$ and $P e / A$ are theoretically equivalent, it is often much easier to compute $P e^{*}$ than to compute $P e / A$.

Finally, let us note the similarity between the present $P e^{*}=\eta \eta_{n} \dot{\gamma} / K$ and the dimensionless shear rate introduced by Fagan and Zukoski, $\eta \dot{\gamma} / G$, where $G$ is the elastic modulus of the suspension [43]. These authors noted the collapse of flow curves obtained for charged silica particles in water when they were plotted as a function of this ratio. As an explanation, they invoked the strong role of electrostatic forces and of the microstructure in determining $G$. This argument is similar to the one developed here, with the distinction that $K$ is a normal stress response to the normal compression due to SIM, while $G$ is a response to a tangential strain.

\section{CONCLUSION}

Different approaches exist concerning the continuous modeling of colloidal flows. They differ in the treatment of the mass conservation equation. Some researchers interested by suspensions of macromolecules or small colloids only considered a thermodynamic mass flux given as a Fickian flux with a diffusion coefficient obtained from the GSE relation to account for interparticle colloidal interactions. Other researchers concerned by the modeling of large colloids in flows with large shear rates considered only a shear-induced mass flux due to hydrodynamic interactions between particles. In a general flow, both ingredients are a priori necessary, but it can be anticipated that the thermodynamic and shear-induced fluxes will dominate at low and high shear rates, respectively. It is therefore of practical interest to determine a priori if both types of fluxes have to be implemented in a flow solver. The central question driving the developments of this article was: Can we predict, with simple tools, whether colloidal and hydrodynamic effects are to be expected for prescribed physicochemical and hydrodynamic conditions?

To answer this question, we considered the fully developed channel flow problem for charge-stabilized colloidal suspensions by adapting the SBM of Frank et al. [15]. In this approach, both thermodynamic and hydrodynamic fluxes are modeled. Simulations at various interaction ranges, interaction strengths, and hydrodynamic conditions enabled a phase diagram to be drawn in the $\left(P e, Z l_{B} / a, \kappa a\right)$ space showing regions dominated by colloidal or hydrodynamic effects. The boundary between these domains was found to have a nonintuitive shape, which we could model successfully with a simple stress balance. The main conclusions of this work were deduced from this diagram: (i) the Péclet number alone is 
not sufficient to characterize the flow regime; (ii) a more relevant rescaled Péclet number $P e^{*}$ can be defined as the ratio of a viscous stress scale $\eta \eta_{n}\langle\dot{\gamma}\rangle$ to a thermodynamic stress scale $K$ in order to identify the phase boundary as $P e^{*} \sim 1$; (iii) the thermodynamic stress scale involved in this number is the bulk modulus $K=\phi \partial \Pi / \partial \phi$ deriving from the osmotic pressure at equilibrium here, which has to be modeled carefully to account for subtle physicochemical effects that can influence the flow regime, such as ion condensation.

Although the thermodynamics considered in this article were related to electrostatic interactions, it is worth underlining that the rescaled Péclet number used here can be extended to other types of colloidal interactions. The only requirement is to compute the osmotic pressure, which is often done with the integral equation theory and pair potentials adapted to the type of interaction under consideration.

The SBM strategy employed here is believed to be robust in the present simulations with $P e^{*} \sim 1$. It would, however, require significant improvements to model colloidal flows at $P e^{*}>1$. In these conditions, the thermodynamic stress might not be isotropic anymore and should depend on the microstructure imposed by the shear flow, while the hydrodynamic stresses would also depend on colloidal interactions. Determining the suspension stress tensor as a function of the volume fraction, shear rate, and range and strength of interactions is a key to further progress in the modeling of concentrated charge-stabilized suspension flows, in particular for nonpairwise additive colloidal interactions. This should now be possible with detailed simulations coupling hydrodynamics and electrostatics at the particle scale.

\section{ACKNOWLEDGMENTS}

The authors wish to warmly thank the anonymous reviewer who suggested the present definition of $P e^{*}$ would be more robust than the one which was initially designed. The authors acknowledge the financial support of the Conseil Régional Midi-Pyrénées through the ECOREV Program.

\section{References}

[1] Morris, J. F., and F. Boulay, "Curvilinear flows of noncolloidal suspensions: The role of normal stresses," J. Rheol. (1978-present) 43(5), 1213-1237 (1999).

[2] Leighton, D., and A. Acrivos, "The shear-induced migration of particles in concentrated suspensions," J. Fluid Mech. 181, 415-439 (1987).

[3] Phillips, R. J., R. C. Armstrong, R. A. Brown, A. L. Graham, and J. R. Abbott, "A constitutive equation for concentrated suspensions that accounts for shear induced particle migration," Phys. Fluids A: Fluid Dyn. (1989-1993) 4(1), 30-40 (1992).

[4] Nott, P. R., and J. F. Brady, "Pressure-driven flow of suspensions: simulation and theory," J. Fluid Mech. 275(1), 157-199 (1994).

[5] Deboeuf, A., G. Gauthier, J. Martin, Y. Yurkovetsky, and J. Morris, "Particle pressure in a sheared suspension: A bridge from osmosis to granular dilatancy,” Phys. Rev. Lett. 102, 108301 (2009).

[6] Schmitt, V., C. M. Marques, and F. M. C. Lequeux, "Shear-induced phase separation of complex fluids: The role of flow-concentration coupling," Phys. Rev. E 52, 4009-4015 (1995).
[7] Besseling, R., L. Isa, P. Ballesta, G. Petekidis, M. E. Cates, and W. C. K. Poon, "Shear banding and flow-concentration coupling in colloidal glasses," Phys. Rev. Lett. 105, 268301 (2010).

[8] Zydney, A. L., and C. K. Colton, "A concentration polarization model for the filtrate flux in cross-flow microfiltration of particulate suspensions," Chem. Eng. Commun. 47(1-3), 1-21 (1986).

[9] Romero, C. A., and R. H. Davis, "Global model of crossflow microfiltration based on hydrodynamic particle diffusion," J. Membr. Sci. 39(2), 157-185 (1988).

[10] Belfort, G., R. H. Davis, and A. L. Zydney, "The behavior of suspensions and macromolecular solutions in crossflow microfiltration," J. Membr. Sci. 96(1), 1-58 (1994).

[11] Bacchin, P., P. Aimar, and V. Sanchez, "Model for colloidal fouling of membranes," AIChE J. 41(2), 368-376 (1995).

[12] Bowen, W. R., and P. M. Williams, "Prediction of the rate of crossflow ultrafiltration of colloids with concentration-dependent diffusion coefficient and viscosity-theory and experiment," Chem. Eng. Sci. 56(10), 3083-3099 (2001).

[13] Bowen, W. R., and P. M. Williams, "Quantitative predictive modelling of ultrafiltration processes: Colloidal science approaches," Adv. Colloid Interface Sci. 134-135, 3-14 (2007). Surface forces: wetting phenomena, membrane separation, rheology. Topical issue in honour of Victor Starov.

[14] Roa, R., E. K. Zholkovskiy, and G. Nagele, "Ultrafiltration modeling of non-ionic microgels," Soft Matter 11, 4106-4122 (2015).

[15] Frank, M., D. Anderson, E. R. Weeks, and J. F. Morris, "Particle migration in pressure-driven flow of a Brownian suspension," J. Fluid Mech. 493(10), 363-378 (2003).

[16] Foss, D. R., and J. F. Brady, "Structure, diffusion and rheology of Brownian suspensions by Stokesian Dynamics simulation," J. Fluid Mech. 407(3), 167-200 (2000).

[17] Batchelor, G. K., "The stress system in a suspension of force-free particles,” J. Fluid Mech. 41(4), 545-570 (1970).

[18] Nott, P. R., E. Guazzelli, and O. Pouliquen, "The suspension balance model revisited," Phys. Fluids (1994-present) 23(4), 043304 (2011).

[19] Brady, J. F., and M. Vicic, "Normal stresses in colloidal dispersions," J. Rheol. (1978-present) 39(3), 545-566 (1995).

[20] Phung, T. N., J. F. Brady, and G. Bossis, "Stokesian Dynamics simulation of Brownian suspensions,” J. Fluid Mech. 313(4), 181-207 (1996).

[21] Morris, J. F., and B. Katyal, "Microstructure from simulated Brownian suspension flows at large shear rate," Phys. Fluids 14(6), 1920-1937 (2002).

[22] Wagner, N. J., and J. F. Brady, "Shear thickening in colloidal dispersions," Phys. Today 62(10), 27-32 (2009).

[23] Russel, W. B., "The rheology of suspensions of charged rigid spheres," J. Fluid Mech. 85(3), 209-232 (1978).

[24] Nazockdast, E., and J. F. Morris, "Effect of repulsive interactions on structure and rheology of sheared colloidal dispersions," Soft Matter $\mathbf{8}$, 4223-4234 (2012).

[25] Mewis, J., and N. J. Wagner, Colloidal Suspension Rheology (Cambridge University, Cambridge, UK, 2012).

[26] Carnahan, N. F., and K. E. Starling, "Equation of state for nonattracting rigid spheres,” J. Chem. Phys. 51(2), 635-636 (1969).

[27] Woodcock, L. V., "Gass transition in the hard-sphere model and Kauzmann's paradox,” Ann. N. Y. Acad. Sci. 371(1), 274-298 (1981).

[28] Pusey, P. N., and W. van Megen, "Properties of concentrated suspensions of slightly soft colloidal spheres," in Physics of Complex and Supermolecular Fluids, edited by S. A. Safran and N. A. Clark (Plenum, New York), pp. 673-698.

[29] Russel, W., D. Saville, and W. Schowalter, Colloidal Dispersions (Cambridge University, Cambridge, UK, 1999). 
[30] Trizac, E., L. Bocquet, M. Aubouy, and H. H. von Grünberg, "Alexander's prescription for colloidal charge renormalization," Langmuir 19(9), 4027-4033 (2003).

[31] Belloni, L., "Ionic condensation and charge renormalization in colloidal suspensions," Colloids Surf. A 140, 227-243 (1998).

[32] Hallez, Y., J. Diatta, and M. Meireles, "Quantitative assessment of the accuracy of the Poisson-Boltzmann cell model for salty suspensions," Langmuir 30(23), 6721-6729 (2014).

[33] Wennerstrom, H., B. Jonsson, and P. Linse, "The cell model for polyelectrolyte systems. Exact statistical mechanical relations, Monte Carlo simulations, and the Poisson-Boltzmann approximation," J. Chem. Phys. 76(9), 4665-4670 (1982).

[34] Alexander, S., P. M. Chaikin, P. Grant, G. J. Morales, P. Pincus, and D. Hone, "Charge renormalization, osmotic pressure, and bulk modulus of colloidal crystals: Theory,” J. Chem. Phys. 80(11), 5776-5781 (1984).

[35] Holm, C., P. Kékicheff, and R. Podgornik, Electrostatic Effects in Soft Matter and Biophysics, NATO Science Series (Springer, Netherlands, 2000).

[36] Semwogerere, D., J. F. Morris, and E. R. Weeks, "Development of particle migration in pressure-driven flow of a Brownian suspension," J. Fluid Mech. 581(6), 437-451 (2007).
[37] Vollebregt, H. M., R. G. M. van der Sman, and R. M. Boom, "Suspension flow modelling in particle migration and microfiltration," Soft Matter 6(24), 6052-6064 (2010).

[38] Persello, J., "Surface and interface structure of silicas," Surfactant Sci. Ser. 90, 297-342 (2000).

[39] Hocine, S., R. Hartkamp, B. Siboulet, M. Duvail, B. Coasne, P. Turq, and J.-F. Dufrêche, "How ion condensation occurs at a charged surface: A molecular dynamics investigation of the stern layer for water-silica interfaces," J. Phys. Chem. C 120, 963-973 (2016).

[40] Trizac, E., M. Aubouy, and L. Bocquet, "Analytical estimation of effective charges at saturation in Poisson-Boltzmann cell models," J. Phys.: Condens. Matter 15(1), S291-S296 (2003).

[41] Dhont, J. K., An Introduction to Dynamics of Colloids (Elsevier, Amsterdam, Netherlands, 1996).

[42] Morris, J., "A review of microstructure in concentrated suspensions and its implications for rheology and bulk flow," Rheol. Acta 48(8), 909-923 (2009).

[43] Fagan, M. E., and C. F. Zukoski, "The rheology of charge stabilized silica suspensions,” J. Rheol. 41(2), 373-397 (1997). 